\title{
Increased common atrioventricular valve tenting is a risk factor for progression to severe regurgitation in patients with a single ventricle with unbalanced atrioventricular septal defect
}

\author{
Chodchanok Vijarnsorn, MD, Nee Scze Khoo, MD, Edythe B. Tham, MD, Timothy Colen, MD, \\ Ivan M. Rebeyka, MD, and Jeffrey F. Smallhorn, MD
}

\begin{abstract}
Objective: Significant atrioventricular valve regurgitation (AVVR) increases mortality in patients with unbalanced atrioventricular septal defects (uAVSDs) and a single ventricle. We tested the hypothesis that abnormal leaflet tethering is associated with progressive AVVR in patients with a single ventricle with uAVSD.
\end{abstract}

\begin{abstract}
Methods: We retrospectively reviewed the initial presentation and prebidirectional cavopulmonary anastamosis echocardiograms of 46 consecutive patients with uAVSD with single ventricle palliation. AVVR was graded as moderate to severe if the sum of vena contracta width to dominant valve annulus ratio was $\geq 0.33$. We measured tenting height, annular to leaflet angle and annular diameter, indexed to patient size where appropriate. Multivariate analysis of variables to predict progressive AVVR was performed.
\end{abstract}

Results: At follow-up of $3.3 \pm 2.4$ years, 24 patients had mild AVVR (Group A) and 22 had moderate to severe AVVR. Overall mortality was $6 \%$, whereas 10 had valve repair/replacement surgery. Of 22 patients with severe AVVR at follow-up, 9 had severe AVVR at initial presentation (Group B), whereas 13 had mild AVVR at presentation but developed severe AVVR at their prebidirectional cavopulmonary anastamosis echocardiogram (Group C). Group A patients had a smaller tenting height at initial presentation compared with patients in Group $\mathrm{B}$ and Group $\mathrm{C}$, and also had early progressive reduction of indexed tenting height $(P<.01)$. An absolute tenting height $>6 \mathrm{~mm}$ (odds ratio, 6.6; 95\% confidence interval, 1.1-39.0; $P=.03$ ) at the initial echocardiogram was identified as an independent predictor of subsequent severe AVVR.

Conclusions: Early leaflet tethering is predictive of subsequent AVVR in patients with a single ventricle with uAVSD. Patients with competent AVV had progressive reduction in the degree of leaflet tethering, whereas patients with AVVR did not. This may represent an important adaptive process to maintain valve competency in uAVSD. (J Thorac Cardiovasc Surg 2014;148:2580-8)

Unbalanced atrioventricular septal defect (UAVSD) is an uncommon congenital heart defect, comprising $<1 \%$ of all congenital heart disease. ${ }^{1,2}$ It is a nonhomogeneous lesion with right ventricular dominance being more frequent $^{2-4}$ and it is frequently associated with heterotaxy. Those patients who are not deemed suitable for a biventricular repair are surgically palliated by staged operations to a total cavopulmonary connection. uAVSD with single ventricle circulation has a particularly high morbidity and mortality rate, ${ }^{5,6}$ more so than seen in patients with classic hypoplastic left heart syndrome

From the Faculty of Medicine and Dentistry, Stollery Children's Hospital, University of Alberta, Edmonton, Alberta, Canada.

C. Vijarnsorn received a scholarship for a fellowship training in University of Alberta from the Faculty of Medicine, Siriraj Hospital, Mahidol University, Bangkok, Thailand.

Disclosures: Authors have nothing to disclose with regard to commercial support.

Received for publication April 4, 2014; revisions received July 26, 2014; accepted for publication Aug 3, 2014; available ahead of print Sept 12, 2014.

Address for reprints: Chodchanok Vijarnsorn, MD, Siriraj Hospital, 2 Wanglang Rd, Bangkok noi, Bangkok, Thailand 10700 (E-mail: cvijarnsorn@yahoo.com).

0022-5223/\$36.00

Copyright (c) 2014 by The American Association for Thoracic Surgery

http://dx.doi.org/10.1016/j.jtcvs.2014.08.005
(HLH). ${ }^{7,8}$ The presence of associated atrioventricular valve regurgitation (AVVR) is frequent in UAVSD with up to $15 \%$ to $30 \%$ requiring surgical intervention; it is also an independent predictor of mortality. ${ }^{9-11}$

Leaflet prolapse and tethering of the mitral valve, tricuspid valve (TV), and left atrioventricular valve postAVSD repair have been previously identified as important mechanisms of valve failure. ${ }^{12-15}$ In a more recent study, ${ }^{16}$ we noted that prolapse as a mechanism of TV regurgitation in HLH is a later phenomenon, with TV tethering being the predominant feature in neonates with HLH who subsequently developed severe tricuspid regurgitation. With this background, we hypothesized that the presence of leaflet tethering in the common atrioventricular valve (AVV) may be an important precursor to subsequent severe regurgitation in patients with an uAVSD. ${ }^{17-19}$

\section{METHODS \\ Data Sources and Variables}

Following institutional ethics board approval the echocardiogram of all patients with uAVSD between January 2003 and July 2012 was identified from our electronic database. The inclusion criterion was an identifiable echocardiogram at the initial evaluation before any surgical intervention at Stollery Children's hospital. Patients who subsequently underwent 


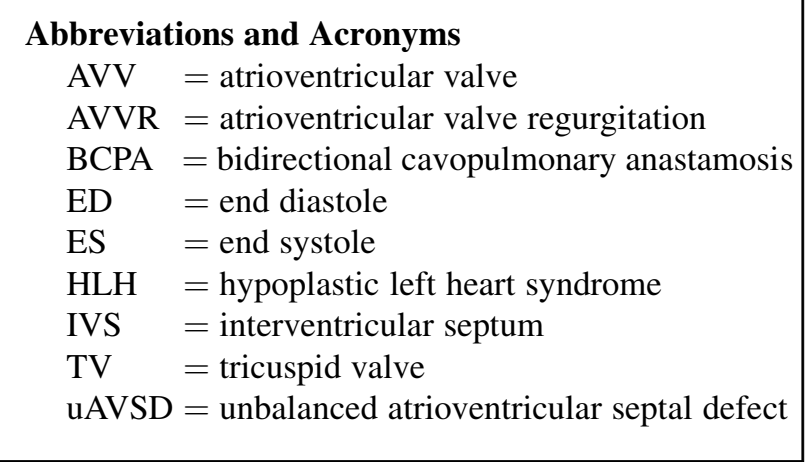

biventricular repair at any stage were excluded. Demographic, anatomic, and procedural variables (including date of birth, weight, height, body surface area, presence of isomerism, presence of trisomy 21 , dominant ventricular morphology, presence of pulmonary or aortic outflow obstruction, surgical procedures, and date of surgical procedure), and current status (alive or deceased or cardiac transplantation) were recorded.

\section{Two-Dimensional Echocardiographic Measurements}

Patients' 2-dimensional echocardiographic images were analyzed at initial presentation, prebidirectional cavopulmonary anastamosis (BCPA), post-BCPA, and at the most recent follow-up.

Measurements were made by a single observer (C.V.) on Q-Lab (Phillips Medical Systems, Andover, Mass) and they included total common atrioventricular valve annular diameter in end diastole (ED), dominant valve annulus in $\mathrm{ED}$ and nondominant valve annulus in $\mathrm{ED}$, with indexing to body surface area where appropriate. Degree of AVVR was defined as being moderate to severe if the sum of vena contracta widths to dominant valve annulus ratio in the 4 -chamber view was $\geq 0.33$. The parameters of leaflet tethering measured were:

1) Tenting height to common valve, defined as the distance between leaflet coaptation and the common valve annular plane in ED and end-systole (ES);

2) Tenting height to interventricular septum (IVS), defined as the distance from common valve annular plane to crest of the IVS in ED and ES;

3) Annular to leaflet angle, defined as the angle between the annular plane to the nadir of leaflet coaptation of both the nondominant and dominant valve in ES; and

4) Annular to IVS angle, defined as the angle between the annular plane to the crest of the IVS of both the nondominant and dominant valve in ES (Figure 1).

\section{Data Analysis}

Patients' baseline characteristics and outcomes were summarized using descriptive statistics. Normally distributed data were presented as the mean \pm standard deviation or, in cases where the distribution was not normal, as median with range. The cumulative prevalence of moderate to severe AVVR, requirement of valve repair, cardiac transplantation, and mortality in all patients were reported.

The patients were divided into 3 groups. Group A included those without or with only mild AVVR at presentation and follow-up, Group B included patients with moderate to severe AVVR at initial presentation and at follow-up, and Group C included patients with mild AVVR at initial presentation but moderate to severe AVVR at the pre-BCPA echocardiogram. $\chi^{2}$ tests were used to compare discrete data between groups; in those cases where the expected cell count was $<5$, the Fisher exact test was used. To identify the baseline characteristics associated with outcome, univariate analysis was evaluated and multivariate models were subsequently applied.
Initially, candidate variables showing a possible association with prognosis by univariate analysis $(P<.05)$ were considered starting with the most significant variable. Significant variables were identified by stepwise regression at the $P<.05$ level. Risk ratios were expressed as odds ratios (ORs) with associated $95 \%$ confidence intervals (CIs). Finally, patients who achieved BCPA operation were explored with regard to progression of tenting height and angle change over time. Differences at each echocardiogram time point were compared between the groups with analysis of variance and Dunnett 2-sided post hoc test between Group A and Group C. The statistic analysis was performed with SPSS 13.0 for Windows (IBM-SPSS Inc, Armonk, NY).

\section{RESULTS}

Fifty-nine patients were identified, of whom 13 were excluded ( 9 underwent biventricular repair and in 4 others the initial echocardiogram could not be retrieved). Hence, 46 patients were eligible for the analysis (Figure 2).

\section{Prevalence of Moderate to Severe AVVR, Valve Surgery, and Clinical Outcomes}

Table 1 summarizes demographics and outcomes at $3.3 \pm$ 2.4 years of follow-up. The majority of patients had isomerism $(70 \%)$ and a dominant right ventricle $(76 \%)$ and at the time of latest follow-up 50\% had total cavopulmonary circulation (Fontan or Kawashima procedure).

Moderate to severe AVVR was present in 22 patients $(48 \%)$ during the follow-up period with 10 undergoing AVV surgery ( 9 valve repair and 1 valve replacement). Valve surgical intervention occurred at a mean age of 2.1 \pm 1.5 years. Three patients had their repair in association with BCPA, 2 at Fontan operation, 1 at Kawashima shunt, and 1 at pulmonary artery banding. Of the remaining 3 patients, 2 had valve repair after BCPA and 1 after Fontan operation. Valve repair was successful in 4 patients with less than mild postoperative regurgitation, whereas 6 had ongoing moderate to severe AVVR. One of these patients died from multiorgan failure after repair, another required cardiac transplantation, and a third died 18 months postoperatively from cardiac failure, whereas the other 3 are still alive.

Of 12 patients with moderate to severe AVVR who did not undergo valve surgery, 8 had no change in severity, 1 patient underwent cardiac transplantation due to severe ventricular dysfunction, and 3 had improvement in their AVVR severity after the BCPA.

\section{Analysis of Nonechocardiographic Risk Factors for Development of Moderate to Severe AVVR at Follow-up}

Demographics and clinical status during a follow-up period of $3.3 \pm 2.4$ years is summarized in Table 2. Using a predictive model, clinical variables (see Table 1) were not different between patients with and without moderate to severe AVVR. Univariate risk analysis did not find any association between clinical variables listed with 

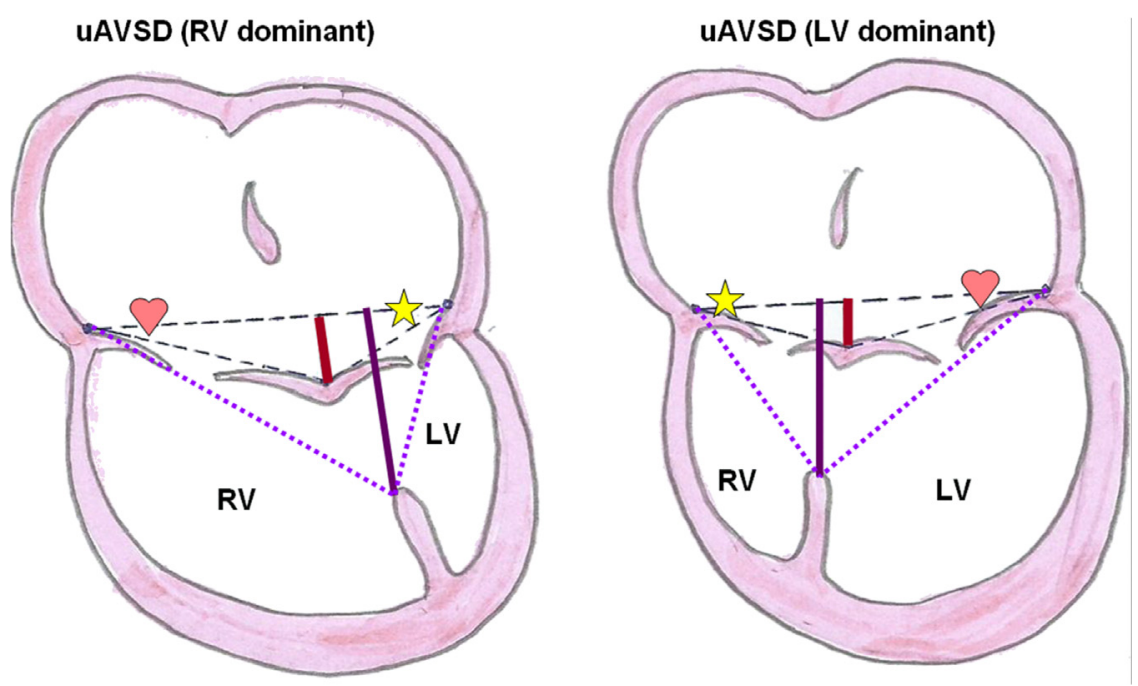

FIGURE 1. Method of echocardiographic measurements: tenting height of common valve (red line), tenting height from interventricular crest (purple line), annular to leaflet angle of dominant ventricle $(\mathrm{O})$, and annular to leaflet angle of nondominant (or hypoplastic) ventricle ( $\hat{\xi})$. Annular to interventricular septum (IVS) angle, defined as the angle between the annular plane to the crest of the IVS. $R V$, Right ventricle; $L V$, left venticle; $u A V S D$, unbalanced atrioventricular septal defect.

subsequent moderate to severe AVVR or valve surgery. Only moderate or greater AVVR at initial presentation was predictive of follow-up moderate to severe AVVR (OR, 2.8; 95\% CI, 1.4-8.1; $P<.005$ ).

\section{Echocardiographic Parameters at Initial Assessment Before Any Surgical Intervention}

Patients who had moderate to severe AVVR at follow-up were older at their initial echocardiogram and had larger body size (Table 3 ). They had greater indexed tenting height to common valve (ED) $\left(28.6 \pm 12.6\right.$ vs $21.1 \pm 6.8 \mathrm{~mm} / \mathrm{m}^{2}$; $P=.02$ ) than patients with mild or less AVVR (Group A). The indexed tenting height to the IVS and the annular to leaflet angle were not different. When we excluded the 9 patients with moderate to severe AVVR at presentation (Group B), both the absolute and indexed tenting height to common valve (ED) remained increased when compared with Group A (mild or no AVVR at presentation), whereas the indexed tenting height to the IVS was not different.

\section{Echocardiographic Parameters at Pre-BCPA Assessment}

At pre-BCPA assessment, patients who had moderate to severe AVVR during follow-up continued to demonstrate differences identified at initial echocardiogram (ie, increased absolute and indexed tenting height to common valve at $\mathrm{ED}$ ) (Table 3). In addition, the indexed tenting height to common valve at ES, indexed nondominant valve diameter, indexed total common valve annulus, and the annulus to leaflet angle in the nondominant valve were increased. These differences remained even when the 9 patients with moderate to severe AVVR at presentation (Group B) were excluded.

\section{Univariate Analysis for Echocardiographic Parameters Association With Progression to Moderate to Severe AVVR and Risk Analysis for Association With AVV Surgery}

Univariate analysis showed that the presence of moderate AVVR at the first presentation, the initial echocardiogram of an indexed tenting height to common valve at ED $>31$ $\mathrm{mm} / \mathrm{m}^{2}$ (OR, 10.7; 95\% CI, 1.2-96.2; $P=.01$ ) and absolute tenting height to common valve at $\mathrm{ED}>6 \mathrm{~mm}(\mathrm{OR}, 9.2$; $95 \% \mathrm{CI}, 1.7-48.8 ; P=.004)$ were associated with subsequent moderate to severe AVVR. Pre-BCPA indexed tenting height to common valve at $\mathrm{ED}>21.5 \mathrm{~mm} / \mathrm{m}^{2}$ (OR, 4.8; $95 \% \mathrm{CI}, 1.4-17.3 ; P=.01)$, indexed tenting height to common valve at $\mathrm{ES}>12 \mathrm{~mm} / \mathrm{m}^{2}$ (OR, 10.2; $95 \%$ CI, 2.5-41.7; $P=.001$ ), and annulus to leaflet angle of the nondominant valve at $\mathrm{ES}>30^{\circ}(\mathrm{OR}, 8.0 ; 95 \% \mathrm{CI}, 1.8-34.9 ; P=.003)$ were also associated with progression to moderate to severe AVVR.

Likewise, risk analysis for AVV surgery identified the following variables, moderate or greater AVVR (OR, 8.0; $95 \% \mathrm{CI}, 1.5-40.3 ; P=.006$ ), indexed tenting height to common valve at ED $>31 \mathrm{~mm} / \mathrm{m}^{2}$ (OR, 5.3; 95\% CI, 1.1-27.4; $P=.03$ ) and absolute tenting height to common valve at $\mathrm{ED}>6 \mathrm{~mm}$ (OR, 7.5; 95\% CI, 1.6-34.9; $P=.006)$ at initial echocardiogram and indexed tenting height to common valve at $\mathrm{ED}>21.5 \mathrm{~mm} / \mathrm{m}^{2}$ (OR, 6.0; $95 \% \mathrm{CI}, 1.0-32.5 ; P=.02$ ), indexed tenting height to common valve at $\mathrm{ES}>12 \mathrm{~mm} / \mathrm{m}^{2}$ (negative relative risk, 0.6 ; $95 \%$ CI, $0.4-0.8 ; P=.001$ ), and annulus to leaflet angle 


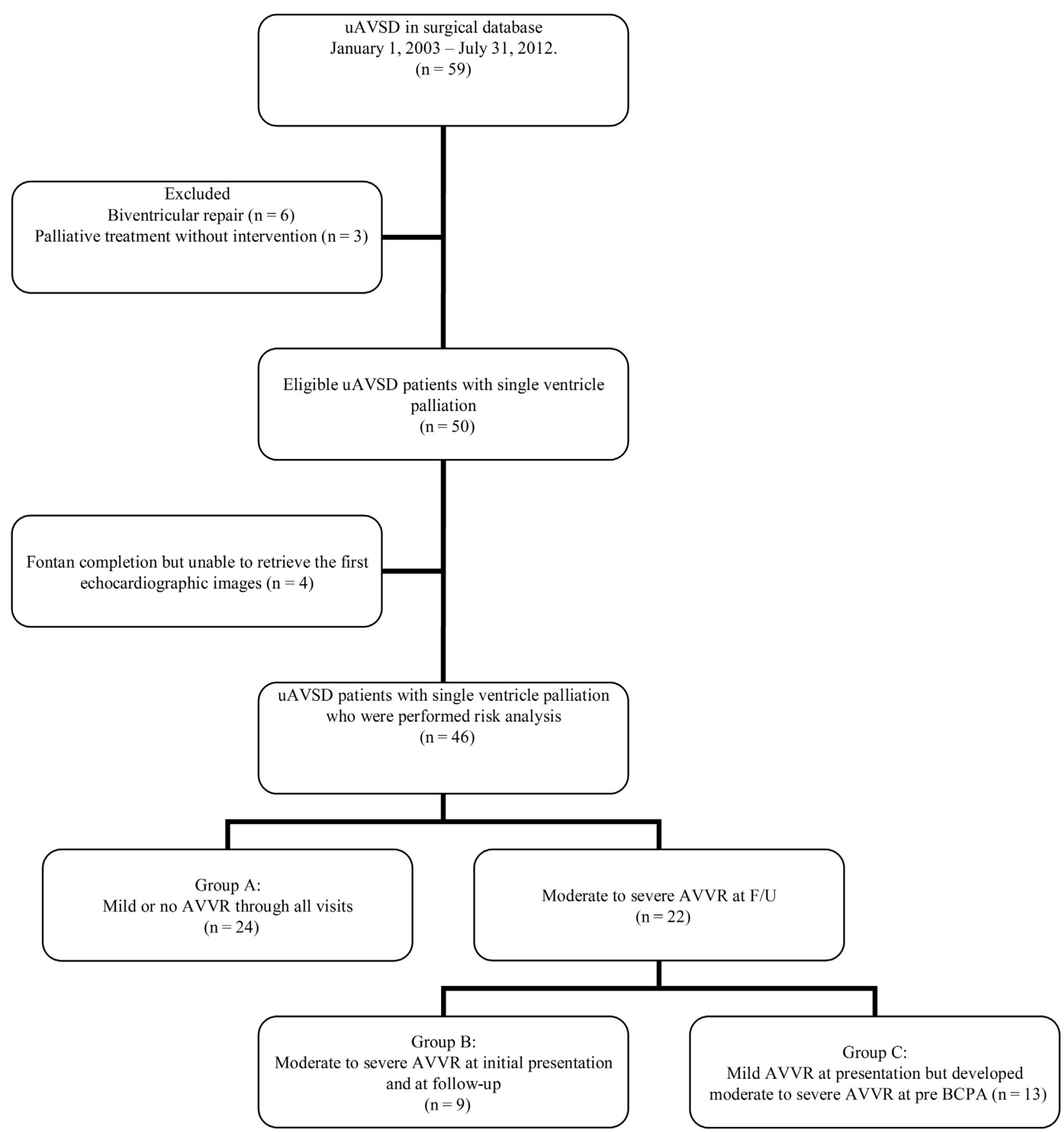

FIGURE 2. Flow chart of participants in the study. $u A V S D$, Unbalanced atrioventricular septal defect; $A V V R$, atrioventricular valve regurgitation; $F / U$, follow-up; BCPA, bidirectional cavopulmonary anastamosis.

of the nondominant valve at $\mathrm{ES}>30^{\circ}(\mathrm{OR}, 4.3 ; 95 \% \mathrm{CI}$, $1.0-18.1 ; P=.04)$ at the pre-BCPA echocardiogram, as risk factors.

\section{Multivariate Analysis for Predictors of Progression to Moderate to Severe AVVR}

To eliminate the first covariate of the presence of moderate AVVR at the first presentation, only patients with mild
AVVR at follow-up (Group A) and patients with initial mild AVVR who progressed to moderate to severe AVVR (Group C) were compared on the predictive model (Table 4). All parameters in the univariate model still appeared to be risk factors of subsequent moderate to severe AVVR on the first step logistic regression. However only absolute tenting height to common valve at $\mathrm{ED}$ at the initial echocardiogram $>6 \mathrm{~mm}$ was an independent predictor. 

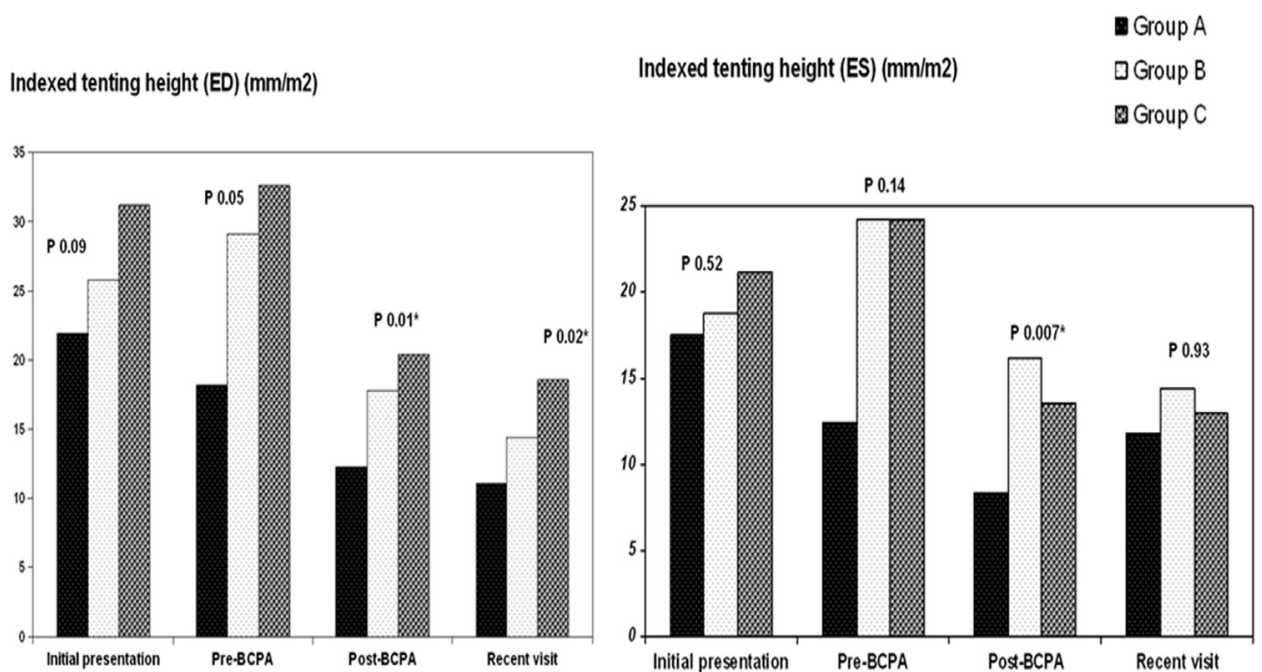

FIGURE 3. Comparison of mean tenting height index (end diastole $[E D]$ and end systole $[E S]$ ) at a first presentation, before bidirectional cavopulmonary anastomosis (pre-BCPA), post-BCPA, and at the most recent visit between Group A: patients without or with only mild atrioventricular valve regurgitation $(A V V R)(n=17)$, Group B: patients with moderate to severe AVVR at initial presentation and at follow-up $(n=6)$, and Group C: patients with mild AVVR at presentation but who developed severe moderate to severe AVVR at their pre-BCPA echocardiogram $(n=9)$ using analysis of variance statistics.

When pre-BCPA echocardiographic variables were included in the analysis, indexed tenting height to common valve at $\mathrm{ES}>12 \mathrm{~mm} / \mathrm{m}^{2}$ was also identified.

TABLE 1. Baseline patient characteristics, staged palliation, and clinical outcomes at follow-up $(\mathrm{N}=46)$

\begin{tabular}{lc}
\hline \multicolumn{1}{c}{ Characteristic } & Result \\
\hline Age at follow-up (y) & $3.3 \pm 2.4$ \\
Male gender & $26(56.5)$ \\
Diagnosis of heterotaxy & $33(69.5)$ \\
$\quad$ Right isomerism & $21(45.6)$ \\
Left isomerism & $11(13.9)$ \\
Presence of trisomy 21 & $3(6.5)$ \\
Dominant ventricle & \\
$\quad$ Right & $36(78.2)$ \\
$\quad$ Left & $10(21.7)$ \\
Presence of aortic obstruction & $11(23.9)$ \\
Presence of pulmonary outflow obstruction & $31(67.3)$ \\
Achieved single ventricle palliation stage & \\
Fontan operation & $18(39.1)$ \\
Kawashima operation & $5(10.9)$ \\
Bidirectional cavopulmonary anastomosis & $11(23.9)$ \\
Others (eg, Norwood operation, arterioportal shunt, & $12(26.1)$ \\
$\quad$ pulmonary artery banding, or repair pulmonary veins) & \\
Clinical outcome & \\
Deceased & $3(6.5)$ \\
Required cardiac transplantation & $2(4.3)$ \\
Presence of moderate to severe AVVR during follow-up & $22(47.8)$ \\
Required valve surgery & $10(21.7)$ \\
Ongoing moderate to severe AVVR to the recent follow-up & $11(26.8)$ \\
$\quad$ in 2012 (excluded 2 heart transplant patients and 3 \\
$\quad$ deceased patients) & \\
\hline
\end{tabular}

Values are expressed as mean \pm standard deviation or $\mathrm{n}(\%) . A V V R$, Atrioventricular valve regurgitation.

\section{Progressive Change in Indexed Tenting Height}

Indexed tenting height to common valve in ED and ES were analyzed in 32 patients with echocardiograms at initial presentation, pre-BCPA, post-BCPA, and the most recent visit (including post-Fontan operation; Figure 3). There were 17 patients in Group A, 6 patients in Group B, and 9 patients in Group C. The indexed tenting height to common valve at ED in Group A were consistently smaller than Group $\mathrm{C}$ at each time point from pre-BCPA until the most recent follow-up, whereas at initial presentation there was only a trend $(P=.09)$. Group A patients also showed progressive reduction of indexed tenting height to common valve at ED at each subsequent stage (initial presentation $21.9 \pm 6.3 \mathrm{vs}$ pre-BCPA $18.2 \pm 6.2 ; P=.02$ and pre-BCPA vs postBCPA $12.4 \pm 6.0 ; P=.002)$ whereas patients in Group C did not (initial presentation $31.3 \pm 13.9$ vs pre-BCPA 32.7 $\pm 26.0 ; P=.88$ and pre-BCPA vs post-BCPA $20.4 \pm 8.3$; $P=.12$ ). Five patients in Group B and 5 patients in Group $C$ had surgical valve repair. Nine of 10 patients had reduction of AVV tenting height, which would skew longitudinal findings of Group A from Group C toward nonsignificance.

\section{DISCUSSION}

Severe AVV regurgitation in patients with uAVSD with single ventricle physiology carries significant morbidity and mortality, so much so that primary neonatal transplantation may be considered a better alternative to single ventricle palliation. However the literature on the mechanisms and predictors of AVV failure in UAVSD is limited. The significant findings of our study confirm previous observations that moderate or greater AVVR at the initial echocardiogram predicts persistent regurgitation at 
TABLE 2. Baseline patient characteristics and outcomes in patients with a single ventricle with unbalanced atrioventricular septal defect in predictive analysis $(\mathbf{N}=\mathbf{4 6})$

\begin{tabular}{|c|c|c|c|c|}
\hline Characters and variables & $\begin{array}{l}\text { All patients } \\
(\mathbf{N}=46)\end{array}$ & $\begin{array}{l}\text { Patients without or } \\
\text { only minimal AVVR } \\
(\text { Group A) }(\mathbf{n}=\mathbf{2 4})\end{array}$ & $\begin{array}{l}\text { Patients who } \\
\text { experienced moderate to } \\
\text { severe AVVR }(n=22) \\
\end{array}$ & $P$ value \\
\hline Age at earliest echocardiogram (d) & $26.8 \pm 67.7$ & $3.9 \pm 6.2$ & $52.2 \pm 92.2$ & .02 \\
\hline Age at follow-up (y) & $3.3 \pm 2.4$ & $3.0 \pm 2.1$ & $3.7 \pm 2.7$ & .29 \\
\hline Male gender & $26(56.5)$ & $13(54.1)$ & $13(59.0)$ & .73 \\
\hline Diagnosis of heterotaxy & $33(71.7)$ & $19(79.1)$ & $13(59.0)$ & .13 \\
\hline Presence of trisomy 21 & $3(6.5)$ & $0(0)$ & $3(13.6)$ & .06 \\
\hline \multicolumn{5}{|l|}{ Dominant ventricle } \\
\hline Right & $36(78.2)$ & $20(83.3)$ & $16(72.8)$ & .38 \\
\hline Left & $10(21.7)$ & $4(16.7)$ & $6(27.2)$ & .42 \\
\hline Presence of aortic obstruction & $11(23.9)$ & $3(12.5)$ & $8(36.0)$ & .06 \\
\hline Presence of pulmonic outflow obstruction & $31(67.3)$ & $12(50)$ & $10(45.4)$ & .75 \\
\hline Presence of at least moderate AVVR at first presentation & $9(19.5)$ & $0(0)$ & $9(40.9)$ & $<.005$ \\
\hline Presence of restricted ventricular septal defects & $10(21.7)$ & $3(12.5)$ & $7(31.8)$ & .13 \\
\hline Presence of restricted atrial septal defects & $1(2.1)$ & $1(4.1)$ & $0(0)$ & .33 \\
\hline Single papillary muscle & $4(8.6)$ & $2(8.3)$ & $2(9.0)$ & .92 \\
\hline \multicolumn{5}{|l|}{ Clinical outcome } \\
\hline Staged Fontan completion & $18(39)$ & $11(45.8)$ & $7(31.8)$ & .33 \\
\hline Deceased & $3(6.5)$ & $1(4.1)$ & $2(9.0)$ & .49 \\
\hline Required transplant & 2(4.) & $0(0)$ & $2(9.0)$ & $<.005$ \\
\hline Required valve repair & $10(21.7)$ & $0(0)$ & $10(45.4)$ & $<.005$ \\
\hline $\begin{array}{l}\text { Ongoing moderate to severe AVVR to the recent } \\
\text { follow-up excluded } 2 \text { heart transplant patients and } \\
3 \text { deceased patients) }\end{array}$ & $11(23)$ & $0(0)$ & $11(50)$ & $<.005$ \\
\hline
\end{tabular}

Values are given as mean \pm standard deviation or $\mathrm{n}(\%)$. AVVR, Atrioventricular valve regurgitation.

follow-up. ${ }^{9,20}$ More importantly we identified that increased tenting height to common valve at ED in the initial echocardiogram and increased tenting height to common valve at ES in the pre-BCPA echocardiogram are independent predictors of severe AVVR in cases with no or mild AVVR at presentation.

Not only did patients who maintained valve competency during follow-up have less tenting height to common valve (a measure of leaflet tethering), but they also demonstrated progressive reduction in the tenting height over time, in contrast to those who developed moderate to severe AVVR. This finding emphasizes the importance of early leaflet tethering as a mechanism for AVV failure in uAVSD and highlights the presence of previously unrecognized leaflet tethering in a subset of uAVSD with a competent $\mathrm{AVV}$ at presentation.

\section{Single Ventricle Palliation Outcomes and Strategies for Progressive AVVR in uAVSD}

Literature focusing on survival and outcomes of UAVSD with single ventricle circulation are sparse. Before 1990, overall mortality of uAVSD surgery was high, ranging from $57 \%$ to $100 \%$. $^{21}$ With improvement in operative and medical strategies over the past 2 decades, $5,6,12$ hospital survival of BCPA procedure has improved
$(91 \%){ }^{22}$ However long-term survival remained modest at $50 \%$ in a cohort of 35 patients with a median follow-up of 25 months $^{23}$ and $66 \%$ at 30 months in second cohort of 22 patients. ${ }^{21}$ These survival data are considerably lower than current HLH cohorts. ${ }^{7,8}$

To our knowledge, our report is on 1 of the larger cohorts of uAVSD with functional single ventricle circulation. In addition to mortality, we report on the prevalence of moderate to severe AVVR and valve intervention. Moderate to severe AVVR was prevalent $(48 \%)$ in our cohort, with an overall mortality of $6 \%$ and cardiac transplantation in $4 \%$ of patients during a median follow-up 31 months (range, 3 months- 8.6 years). The influence of moderate or severe AVVR on morbidity is significant with $48 \%$ requiring medical therapy and 1 patient progressing to ventricular pump failure and subsequent cardiac transplantation.

The strategy of ventricular unloading following BCPA operation, although reported to have greater effect on reduction of AVVR in other types of single ventricle hearts, was only effective in $12 \%$ of our patients with moderate or severe AVVR, suggesting this strategy may not be as effective in infants with uAVSD. ${ }^{10}$ Twenty percent of our total cohort underwent $\mathrm{AV}$ valve repair surgery ( $40 \%$ of patients within the moderate to severe AVVR group) with a satisfactory outcome in half where postoperative regurgitation is 
TABLE 3. Morphologic variables evaluated by initial echocardiography $(N=46)$ between Group A: patients without or with only mild atrioventricular valve regurgitation $(A V V R)(n=24)$, Group B: patients with moderate to severe AVVR at initial presentation and at follow-up $(n=9)$, and Group C: patients with mild AVVR at presentation but developed severe AVVR at their prebidirectional cavopulmonary anastomosis echocardiogram $(\mathbf{n}=\mathbf{1 3})$

\begin{tabular}{|c|c|c|c|c|c|}
\hline Characters and variables & $\begin{array}{c}\text { Overall } \\
(N=46)\end{array}$ & $\begin{array}{c}\text { Group A } \\
(n=24)\end{array}$ & $\begin{array}{c}\text { Patients with } \\
\text { moderate to severe } \\
\text { AVVR }(n=22) \\
\end{array}$ & $\begin{array}{c}\text { Group B } \\
(\mathbf{n}=\mathbf{9}) \\
\end{array}$ & $\begin{array}{c}\text { Group C } \\
(n=13)\end{array}$ \\
\hline \multicolumn{6}{|l|}{ Initial echocardiography } \\
\hline Body surface area & $0.21 \pm 0.03$ & $0.20 \pm 0.02$ & $0.23 \pm 0.04 *$ & $0.23 \pm 0.04$ & $0.22 \pm 0.04$ \\
\hline Dominant valve annulus index $\left(\mathrm{mm} / \mathrm{m}^{2}\right)$ & $83.1 \pm 21.9$ & $82.7 \pm 25.4$ & $83.5 \pm 17.8$ & $79.0 \pm 12.6$ & $86.7 \pm 20.6$ \\
\hline Nondominant valve annulus index $\left(\mathrm{mm} / \mathrm{m}^{2}\right)$ & $39.1 \pm 15.4$ & $36.7 \pm 13.1$ & $41.7 \pm 17.6$ & $44.8 \pm 18.9$ & $39.6 \pm 17.1$ \\
\hline Total common valve annulus index $\left(\mathrm{mm} / \mathrm{m}^{2}\right)$ & $110.4 \pm 25.8$ & $109.3 \pm 26.8$ & $111.7 \pm 25.2$ & $110.2 \pm 31.5$ & $112.7 \pm 21.1$ \\
\hline Tenting height to common valve (ED) (mm) & $5.4 \pm 2.8$ & $4.2 \pm 1.4$ & $6.6 \pm 3.4^{*}$ & $7.35 \pm 4.5$ & $6.0 \pm 2.4 \dagger$ \\
\hline Indexed tenting height to common valve (ED) $\left(\mathrm{mm} / \mathrm{m}^{2}\right)$ & $24.7 \pm 10.6$ & $21.1 \pm 6.8$ & $28.6 \pm 12.6^{*}$ & $29.1 \pm 12.8$ & $28.2 \pm 13.1 \dagger$ \\
\hline Tenting height to interventricular septum (ED) (mm) & $9.8 \pm 4.1$ & $8.45 \pm 3.2$ & $11.3 \pm 4.5^{*}$ & $12.2 \pm 5.5$ & $10.6 \pm 3.9$ \\
\hline Indexed tenting height to interventricular septum $(\mathrm{ED})\left(\mathrm{mm} / \mathrm{m}^{2}\right)$ & $46.6 \pm 19.1$ & $43.8 \pm 19.3$ & $49.7 \pm 18.9$ & $50.4 \pm 17.4$ & $49.2 \pm 20.7$ \\
\hline Tenting height to common valve (ES) (mm) & $3.9 \pm 2.0$ & $3.3 \pm 1.2$ & $4.6 \pm 2.4$ & $5.1 \pm 3.2$ & $4.4 \pm 1.9$ \\
\hline Indexed tenting height to common valve (ES) $\left(\mathrm{mm} / \mathrm{m}^{2}\right)$ & $18.3 \pm 7.9$ & $16.5 \pm 5.8$ & $20.2 \pm 9.5$ & $20.3 \pm 9.4$ & $20.1 \pm 9.9$ \\
\hline Tenting height to interventricular septum (ES) (mm) & $8.3 \pm 3.3$ & $7.5 \pm 3.1$ & $9.2 \pm 3.5$ & $9.5 \pm 4.4$ & $9.1 \pm 2.8$ \\
\hline Indexed tenting height to interventricular septum $(\mathrm{ES})\left(\mathrm{mm} / \mathrm{m}^{2}\right)$ & $38.8 \pm 14.8$ & $37.2 \pm 14.7$ & $40.6 \pm 15.0$ & $39.2 \pm 14.9$ & $41.6 \pm 15.6$ \\
\hline Annular to leaflet angle of dominant valve (ES) & $15.4 \pm 8.1$ & $14.6 \pm 7.1$ & $16.2 \pm 9.2$ & $15.0 \pm 6.6$ & $17.1 \pm 10.7$ \\
\hline Annular to interventricular septum angle of dominant valve (ES) & $28.5 \pm 11.5$ & $27.8 \pm 11.7$ & $29.3 \pm 11.5$ & $27.9 \pm 12.1$ & $30.1 \pm 11.7$ \\
\hline Annular to leaflet angle of nondominant valve (ES) & $29.5 \pm 10.5$ & $29.9 \pm 11.1$ & $30.1 \pm 9.8$ & $28.6 \pm 11.2$ & $31.1 \pm 9.1$ \\
\hline Annular to interventricular septum angle of nondominant valve (ES) & $53.1 \pm 13.5$ & $52.5 \pm 14.4$ & $53.5 \pm 13.4$ & $52.1 \pm 17.4$ & $54.4 \pm 9.5$ \\
\hline \multicolumn{6}{|l|}{ Prebidirectional cavopulmonary anastomosis echocardiography } \\
\hline Body surface area & $0.31 \pm 0.08$ & $0.30 \pm 0.06$ & $0.31 \pm 0.08$ & $0.32 \pm 0.13$ & $0.30 \pm 0.08$ \\
\hline Dominant valve annulus index $\left(\mathrm{mm} / \mathrm{m}^{2}\right)$ & $74.1 \pm 19.9$ & $72.0 \pm 18.6$ & $76.4 \pm 21.4$ & $73.3 \pm 23.2$ & $78.5 \pm 20.5$ \\
\hline Nondominant valve annulus index $\left(\mathrm{mm} / \mathrm{m}^{2}\right)$ & $35.6 \pm 14.2$ & $31.1 \pm 14.3$ & $40.3 \pm 12.6^{*}$ & $38.9 \pm 12.3$ & $41.3 \pm 13.1 \dagger$ \\
\hline Total common valve annulus index $\left(\mathrm{mm} / \mathrm{m}^{2}\right)$ & $89.3 \pm 26.9$ & $80.6 \pm 29.2$ & $98.4 \pm 21.4^{*}$ & $95.1 \pm 22.3$ & $100.6 \pm 21.2 \dagger$ \\
\hline Tenting height to common valve (ED) (mm) & $7.2 \pm 4.2$ & $5.5 \pm 1.9$ & $8.8 \pm 5.3^{*}$ & $9.1 \pm 4.7$ & $8.6 \pm 5.9 \dagger$ \\
\hline Indexed tenting height to common valve (ED) $\left(\mathrm{mm} / \mathrm{m}^{2}\right)$ & $23.0 \pm 14.7$ & $18.7 \pm 8.5$ & $28.4 \pm 17.7^{*}$ & $28.0 \pm 8.0$ & $28.7 \pm 12.5 \dagger$ \\
\hline Tenting height to interventricular septum (ED) (mm) & $12.9 \pm 5.1$ & $12.1 \pm 5.2$ & $13.8 \pm 4.9$ & $14.4 \pm 5.0$ & $13.4 \pm 5.1$ \\
\hline Indexed tenting height to interventricular septum $(E D)\left(\mathrm{mm} / \mathrm{m}^{2}\right)$ & $41.5 \pm 17.0$ & $38.1 \pm 19.4$ & $45.1 \pm 13.6$ & $47.2 \pm 16.4$ & $43.5 \pm 11.8$ \\
\hline Tenting height to common valve (ES) (mm) & $5.1 \pm 3.9$ & $3.8 \pm 1.8$ & $6.5 \pm 4.9^{*}$ & $6.6 \pm 3.4$ & $6.4 \pm 5.9$ \\
\hline Indexed tenting height to common valve (ES) $\left(\mathrm{mm} / \mathrm{m}^{2}\right)$ & $17.3 \pm 15.1$ & $13.2 \pm 7.8$ & $21.5 \pm 19.2^{*}$ & $21.1 \pm 10.0$ & $21.9 \pm 14.1 \dagger$ \\
\hline Tenting height to interventricular septum (ES) (mm) & $12.9 \pm 5.1$ & $12.1 \pm 5.2$ & $13.8 \pm 4.9$ & $14.4 \pm 5.0$ & $13.4 \pm 5.1$ \\
\hline Indexed tenting height to interventricular septum $(\mathrm{ES})\left(\mathrm{mm} / \mathrm{m}^{2}\right)$ & $41.5 \pm 17.0$ & $38.1 \pm 19.4$ & $45.1 \pm 13.6$ & $47.2 \pm 16.4$ & $43.5 \pm 11.8$ \\
\hline Annular to leaflet angle of dominant valve (ES) & $14.7 \pm 8.3$ & $12.7 \pm 6.9$ & $16.6 \pm 9.3$ & $20.1 \pm 11.9$ & $14.2 \pm 6.3$ \\
\hline Annular to interventricular septum angle of dominant valve (ES) & $28.3 \pm 12.8$ & $25.8 \pm 11.6$ & $31.4 \pm 13.5$ & $33.8 \pm 13.9$ & $29.8 \pm 13.6$ \\
\hline Annular to leaflet angle of nondominant valve (ES) & $26.7 \pm 11.2$ & $22.3 \pm 8.4$ & $31.3 \pm 12.0^{*}$ & $32.6 \pm 13.8$ & $30.4 \pm 11.1 \dagger$ \\
\hline Annular to interventricular septum angle of nondominant valve (ES) & $51.6 \pm 13.6$ & $49.1 \pm 15.1$ & $54.2 \pm 11.9$ & $55.4 \pm 15.2$ & $53.3 \pm 9.5$ \\
\hline
\end{tabular}

Values are given as mean \pm standard deviation or $\mathrm{n}(\%) . E D$, End diastole; $E S$, end systole; $A V V R$, atrioventricular valve regurgitation. *Statistical difference between patients without or only mild AVVR (Group A) and patient with experienced moderate to severe AVVR $(P<.05)$ by Student $t$ test and Mann-Whitney $U$ test. $\dagger$ Statistical difference between patients without or only mild AVVR (Group A) and patients with mild AVVR at a initial presentation but developed severe AVVR later $(P<.05)$ by Student $t$ test and Mann-Whitney $U$ test.

reduced to less than moderate, suggesting surgical valve repair may be a useful strategy to address AVV failure in some patients with UAVSD.

\section{Mechanisms of Severe AVVR in uAVSD}

The mechanisms of AV valve failure are complex, multifactorial, and incompletely understood, ${ }^{11,24}$ with contributions from abnormalities in annular and leaflet function (eg, annular dilation, prolapse, dysplasia, clefts, and commissural defects) and subvalvular apparatus (increased chordae tethering or papillary muscle position).
Severe AVVR in single ventricle hearts is a risk factor for mortality and appears to be more prevalent in uAVSD than in hearts of other morphology. ${ }^{9,10}$

The results of our study showed that moderate or greater AVVR at initial presentation, and increased leaflet tethering (ie, increased tenting height to common valve) at the initial and pre-BCPA echocardiograms, was associated with subsequent development of severe AVVR in UAVSD patients with single ventricle circulation. In addition, we found progressive reduction of the tenting height in patients who maintained valve competency. These findings led us to 
TABLE 4. Results of multivariable analysis to determine predictors of moderate to severe atrioventricular valve regurgitation (AVVR) in patients with mild AVVR at initial presentation (Group A and Group $\mathbf{C} ; \mathbf{n}=37$ )

\begin{tabular}{|c|c|c|c|c|}
\hline Variable & $\begin{array}{c}\text { Crude odds ratio } \\
(95 \% \text { confidence interval })\end{array}$ & $P$ value* & $\begin{array}{c}\text { Adjusted odds ratio } \\
(\mathbf{9 5} \% \text { confidence interval })\end{array}$ & $P$ value $\dagger$ \\
\hline \multicolumn{5}{|l|}{ First presentation } \\
\hline Indexed tenting height to common valve (ED) $>31 \mathrm{~mm} / \mathrm{m}^{2}$ & $10.2 \ddagger(1.1-104.1)$ & .02 & & \\
\hline Indexed tenting height to common valve $(\mathrm{ES})>18 \mathrm{~mm} / \mathrm{m}^{2}$ & $2.3(0.5-10.9)$ & .25 & & \\
\hline Absolute tenting height to common valve $(\mathrm{ED})>6 \mathrm{~mm}$ & $5.4 †(1.9-57.7)$ & .008 & $6.6 \ddagger(1.1-39.0)$ & .035 \\
\hline \multicolumn{5}{|l|}{ Pre-BCPA operation } \\
\hline Indexed tenting height to common valve (ED) $>21.5 \mathrm{~mm} / \mathrm{m}^{2}$ & $5.1 \doteqdot(1.2-52.4)$ & .02 & & \\
\hline Indexed tenting height to common valve (ES) $>12 \mathrm{~mm} / \mathrm{m}^{2}$ & $7.6 \ddagger(1.5-6.4)$ & .007 & $8.6 \ddagger(1.8-40.7)$ & .006 \\
\hline Annulus to nondominant valve leaflet angle $(\mathrm{ES})>30^{\circ}$ & $7.7 \ddagger(1.5-39.7)$ & .009 & & \\
\hline
\end{tabular}

speculate that leaflet tethering may be an important mechanism for AVV failure in patients with uAVSD. Just as importantly we found a lack of association of common valve annular plane to IVS distance (ie, tenting height to IVS) and AVVR, suggesting that the size of the interventricular communication was not a significant contributor to subsequent AVVR. At this time there is no other comparable study examining mechanisms of AVV failure in uAVSD.

Most of the information on effects of leaflet tethering on valve function arises from the adult literature where mitral valve leaflet tethering (ie, increased tenting height, tenting area, and annular to leaflet angle) is an independent risk factor for progressive AVVR after myocardial infarction. ${ }^{18,19,25,26}$ In congenital heart disease, Bharucha and colleagues $^{12}$ found in a population of patients with complete AVSD that a greater angle of the opening components of the common valve was a marker of moderate to severe AVVR after total correction. This is similar to our finding of greater annular plane to leaflet angle as a risk factor. Our previous work on mechanisms of AVVR in complete AVSD after biventricular repair, identified both tethering from subvalvular apparatus abnormalities and leaflet prolapse as features associated with left AVV failure. ${ }^{14,24,27}$ Perhaps the study with the most striking similarity to ours is our recent investigation on TV function in patients with HLH. Similar to our study, TV leaflet tethering was the only feature associated with subsequent early to midterm TV failure, whereas neonatal TV prolapse was not a predictor in HLH. ${ }^{16}$ In a separate cross-sectional study on TV failure in HLH, we again identified TV leaflet tethering as an important mechanism of valve failure as well as TV prolapse. We noted that leaflet prolapse as a mechanism of tricuspid regurgitation tended to occur in older patients. ${ }^{17}$ These observations, coupled with the increasing evidence of active leaflet remodeling by leaflet expansion as well as chordal lengthening and thickening beneath tethered leaflets, ${ }^{25,26}$ led us to hypothesize that the development of early to midterm AVV failure in single ventricle circulation with increased loading stressors (both volume and pressure) may represent a failure of tethered AVV remodeling by leaflet expansion and chordal lengthening.

\section{Study Limitations}

Although selection bias exists in any retrospective cohort study, we tried to minimize this by collecting parameters according to a strict measurement protocol and excluding cases with incomplete datasets before the risk analysis. We found that a dominant right ventricle, trisomy 21 , and heterotaxy were not associated with subsequent AVVR, which was contrary to some previous series. ${ }^{10}$ This is likely explained by the smaller proportion of patients with trisomy 21 and the higher proportion of dominant right ventricle $(76 \%)$ and heterotaxy $(70 \%)$ coexisting within our cohort that resulted in a lack of discriminatory power for these variables. The analysis of the longitudinal data may have been affected by having 5 patients in Group B and 5 patients in Group $\mathrm{C}$ having had valve surgery. Nine of 10 patients had unchanged or reduced AVV tenting height postoperatively; hence, the likely influence of valve surgery on longitudinal analysis was more likely to skew the results toward us not finding a significant difference between Group A and Group C, because Group A was found to have progressively smaller tenting height, whereas Group $\mathrm{C}$ was found to not significantly change. Nonetheless, the patients who underwent repair may have affected the longitudinal analysis of changes in valve tenting and leaflet angles for Group B and Group C, whereas the trends in Group A would not be affected. The analysis for echocardiographic predictors of valve regurgitation was performed at the initial and preBCPA study, before any valve surgery, and was hence unaffected. Finally, this was a 2-dimensional echocardiography study that limited its study question to leaflet tethering as a mechanism of AVV failure. Even though we identified tethering as a potential mechanism of progressive AVVR in uAVSD, it remains unknown if other components of the AVV may contribute more significantly to early to midterm AVV failure. 


\section{CONCLUSIONS}

Severe AVVR in uAVSD is common and surgical intervention is frequent. Early leaflet tethering is associated with progressive of AVV failure in patients with uAVSD with single ventricle circulation. Patients with competent AVV had progressive reduction in the degree of leaflet tethering, whereas those with AVV regurgitation did not. This progressive change in AVV function may represent an important adaptive process to maintain valve competency in UAVSD.

The authors thank Dr Julaporn Pooliam, Clinical Epidemiology Unit, Office of Research and Development, Faculty of Medicine, Siriraj Hospital, Mahidol University, for providing assistance with the statistics.

\section{References}

1. Samanek M. Congenital heart malformations: prevalence, severity, survival, and quality of life. Cardiol Young. 2000;10:179-85.

2. Geva T, Ayres NA, Pignatelli RH, Gajarski RJ. Echocardiographic evaluation of common atrioventricular canal defects: a study of 206 consecutive patients. Echocardiography. 1996;13:387-400.

3. Cohen MS, Jacobs ML, Weinberg PM, Rychik J. Morphometric analysis of unbalanced common atrioventricular canal using two-dimensional echocardiography. J Am Coll Cardiol. 1996;28:1017-23.

4. Smallhorn JF. Cross-sectional echocardiographic assessment of atrioventricular septal defect: basic morphology and preoperative risk factors. Echocardiography. 2001;18:415-32.

5. Cohen MS, Spray TL. Surgical management of unbalanced atrioventricular canal defect. Semin Thorac Cardiovasc Surg Pediatr Card Surg Annu. 2005;135-44.

6. Mitchell ME, Litwin SB, Tweddell JS. Complex atrioventricular canal. Semin Thorac Cardiovasc Surg Pediatr Card Surg Annu. 2007;32-41.

7. Gaynor JW, Bridges ND, Cohen MI, Mahle WT, Decampli WM, Steven JM, et al. Predictors of outcome after the Fontan operation: is hypoplastic left heart syndrome still a risk factor? J Thorac Cardiovasc Surg. 2002;123:237-45.

8. Tweddell JS, Hoffman GM, Mussatto KA, Fedderly RT, Berger S, Jaquiss RD, et al. Improved survival of patients undergoing palliation of hypoplastic left heart syndrome: lessons learned from 115 consecutive patients. Circulation. 2002;106:I82-9.

9. Ando M, Takahashi Y. Long-term functional analysis of the atrioventricular valve in patients undergoing single ventricle palliation. Ann Thorac Surg. 2011;92:1767-73.

10. Mahle WT, Cohen MS, Spray TL, Rychik J. Atrioventricular valve regurgitation in patients with single ventricle: impact of the bidirectional cavopulmonary anastomosis. Ann Thorac Surg. 2001;72:831-5.

11. Honjo O, Atlin CR, Mertens L, et al. Atrioventricular valve repair in patients with functional single-ventricle physiology: impact of ventricular and valve function and morphology on survival and reintervention. J Thorac Cardiovasc Surg. 2011; 142:326-35.e2.
12. Bharucha T, Sivaprakasam MC, Haw MP, Anderson RH, Vettukattil JJ. The angle of the components of the common atrioventricular valve predicts the outcome of surgical correction in patients with atrioventricular septal defect and common atrioventricular junction. J Am Soc Echocardiogr. 2008;21: 1099-104.

13. Takahashi K, Guerra V, Roman KS, Nii M, Redington A, Smallhorn JF. Threedimensional echocardiography improves the understanding of the mechanisms and site of left atrioventricular valve regurgitation in atrioventricular septal defect. J Am Soc Echocardiogr. 2006;19:1502-10.

14. Takahashi K, Mackie AS, Thompson R, Al-Naami G, Inage A, Rebeyka IM, et al. Quantitative real-time three-dimensional echocardiography provides new insight into the mechanisms of mitral valve regurgitation post-repair of atrioventricular septal defect. J Am Soc Echocardiogr. 2012;25:1231-44.

15. Kutty S, Smallhorn JF. Evaluation of atrioventricular septal defects by threedimensional echocardiography: benefits of navigating the third dimension. $J$ Am Soc Echocardiogr. 2012;25:932-44.

16. Ugaki S, Khoo NS, Ross DB, Rebeyka IM, Adatia I. Tricuspid valve repair improves early right ventricular and tricuspid valve remodeling in patients with hypoplastic left heart syndrome. J Thorac Cardiovasc Surg. 2012;145: 446-50.

17. Takahashi K, Inage A, Rebeyka IM, Ross DB, Thompson RB, Mackie AS, et al. Real-time 3-dimensional echocardiography provides new insight into mechanisms of tricuspid valve regurgitation in patients with hypoplastic left heart syndrome. Circulation. 2009;120:1091-8.

18. Silbiger JJ. Mechanistic insights into ischemic mitral regurgitation: echocardiographic and surgical implications. J Am Soc Echocardiogr. 2011;24:707-19.

19. He S, Fontaine AA, Schwammenthal E, Yoganathan AP, Levine RA. Integrated mechanism for functional mitral regurgitation: leaflet restriction versus coapting force: in vitro studies. Circulation. 1997;96:1826-34.

20. Ten Harkel AD, Cromme-Dijkhuis AH, Heinerman BC, Hop WC, Bogers AJ. Development of left atrioventricular valve regurgitation after correction of atrioventricular septal defect. Ann Thorac Surg. 2005;79:607-12.

21. Mehta S, Hirschfeld S, Riggs T, Liebman J. Echocardiographic estimation of ventricular hypoplasia in complete atrioventricular canal. Circulation. 1979; 59:888-93.

22. van Son JA, Phoon CK, Silverman NH, Haas GS. Predicting feasibility of biventricular repair of right-dominant unbalanced atrioventricular canal. Ann Thorac Surg. 1997;63:1657-63.

23. Owens GE, Gomez-Fifer C, Gelehrter S, Owens ST. Outcomes for patients with unbalanced atrioventricular septal defects. Pediatr Cardiol. 2009;30:431-5.

24. Khoo NS, Smallhorn JF. Mechanism of valvular regurgitation. Curr Opin Pediatr. 2011;23:512-7.

25. Chaput M, Handschumacher MD, Guerrero JL, Holmvang G, Dal-Bianco JP, Sullivan S, et al. Mitral leaflet adaptation to ventricular remodeling: prospective changes in a model of ischemic mitral regurgitation. Circulation. 2009; 120(Suppl):S99-103.

26. Dal-Bianco JP, Aikawa E, Bischoff J, Guerriero JL, Handschumacher MD, Sullivan S, et al. Active adaptation of the tethered mitral valve: insights into a compensatory mechanism for functional mitral regurgitation. Circulation. 2009; 120:334-42.

27. Colen TM, Khoo NS, Ross DB, Smallhorn JF. Partial zone of apposition closure in atrioventricular septal defect: are papillary muscles the clue. Ann Thorac Surg. 2013;96:637-43. 